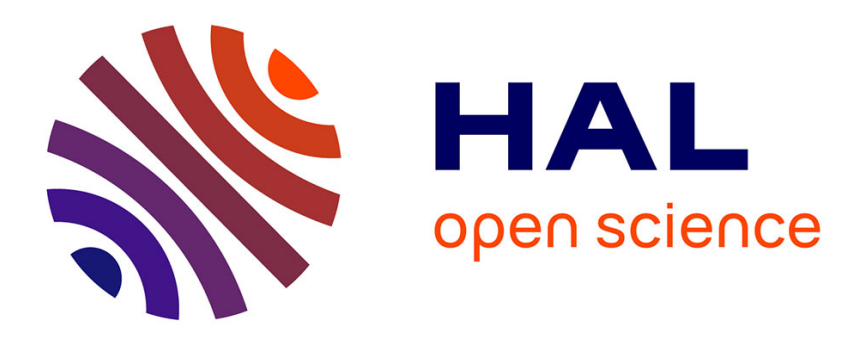

\title{
Effets de la teneur en grain de l'ensilage de maïs sur les performances zootechniques de vaches laitières
}

\author{
J.C. Émile, Y. Barrière
}

\section{To cite this version:}

J.C. Émile, Y. Barrière. Effets de la teneur en grain de l'ensilage de maïs sur les performances zootechniques de vaches laitières. Productions Animales, 1992, 5 (2), pp.113-120. hal-00895967

\section{HAL Id: hal-00895967 \\ https://hal.science/hal-00895967}

Submitted on 1 Jan 1992

HAL is a multi-disciplinary open access archive for the deposit and dissemination of scientific research documents, whether they are published or not. The documents may come from teaching and research institutions in France or abroad, or from public or private research centers.
L'archive ouverte pluridisciplinaire HAL, est destinée au dépôt et à la diffusion de documents scientifiques de niveau recherche, publiés ou non, émanant des établissements d'enseignement et de recherche français ou étrangers, des laboratoires publics ou privés. 
INRA Prod. Anim., $1992,5(2), 113-120$

\section{J.C. ÉMILE, Y. BARRIÈRE}

INRA Station d'Amélioration des Plantes Fourragères

86600 Lusignan
Effets de la teneur en grain de l'ensilage de maïs sur les performances zootechniques de vaches laitières

L'ensilage de maïs est devenu le principal fourrage conservé de l'alimentation des ruminants. Des hybrides caractérisés par une forte productivité en biomasse pourraient permettre d'accroître encore l'intérêt de cette culture pour l'éleveur malgré une teneur en grain plus faible que celle des variétés grain actuelles.

Le maïs ensilé est très largement utilisé en France et en Europe pour l'alimentation des ruminants. Cette plante se caractérise par rapport aux fourrages traditionnels par la juxtaposition en parties presque égales de grains et d'une partie non grain composée de tiges, de feuilles, de spathes et de rafles. La partie grain, composée essentiellement d'amidon, est très digestible par les ruminants (de l'ordre de $90 \%$ ). La digestibilité de la partie non-grain est, quant à elle, plus faible et variable en fonction de sa composition biochimique et des génotypes considérés. La digestibilité de la matière organique des plantes entières est ainsi comprise entre 65 et $76 \%$ (Barrière et al 1992).

Parmi les critères de choix d'une variété pour l'éleveur il y a bien entendu son adaptation aux conditions locales (climat, sol) mais aussi son rendement potentiel en matière

\section{Résumé}

Les performances zootechniques de vaches laitières alimentées avec un ensilage de mais appauvri en épis lors de la récolte ont été comparées à celles de lots témoins recevant l'ensilage normal. En conditions d'apports énergétiques proches de la satisfaction des besoins (3 essais), la réduction de la teneur en grain de 50 à $44 \%$ de la matière sèche n'a eu d'incidence majeure ni sur les quantitées ingérées, ni sur les productions laitières $(22,9$ et $22,8 \mathrm{~kg}$ de lait brut, en moyenne des essais, respectivement pour les lots appauvris et normaux) ou les reprises de poids. Lorsque les apports énergétiques étaient insuffisants (1 essai), on a observé une dégradation des taux butyreux et protéiques. Les estimations de valeurs énergétiques de ces ensilages, fournies par diverses méthodes (composition de la plante, digestibilité in vivo, digestibilité in vitro par des enzymes ou du jus de rumen), ne permettaient pas de prédire ces résultats. L'appauvrissement en épis s'est traduit par une meilleure utilisation par l'animal de la partie non grain du mais $(0,71$ UFL contre 0,66 UFL dans nos conditions). Il semble ainsi possible pour le sélectionneur d'améliorer notablement le rendement en biomasse de cette culture destinée à l'alimentation des vaches laitières en acceptant une diminution de la teneur en grain qui ne serait finalement pas préjudiciable à la qualité. sèche digestible. Barrière et al (1987) ont montré que l'accroissement des rendements en grain dans les dernières décennies ne s'est accompagné d'une amélioration du rendement en biomasse totale que pour les hybrides précoces. Pour les autres, et en particulier pour les hybrides demi-précoces, ce rendement plante entière a eu tendance à stagner, malgré des gains de rendement en grain considérables. L'amélioration du rendement de la partie non grain apparaît donc être une voie à ne pas négliger. Il est actuellement difficile de préciser la teneur en grain optimale d'un ensilage de maïs, préalable à la mise en place de programmes efficaces de sélection. Par sécurité ou facilité, il a été souvent conseillé une teneur en grain maximale, en supposant que la meilleure variété en grain sera la meilleure en ensilage, sans que des mesures sur des animaux n'aient été prises en compte pour étayer cette idée.

Les comparaisons, chez la vache laitière, d'ensilages de maïs en fonction de leur teneur en grain sont rares et les conditions des diverses expérimentations ne sont pas homogènes. Hemken et al (1971) obtiennent des productions laitières semblables avec deux hybrides à teneur en grain différente ( 67 et $56 \%$ de la matière sèche). Lorsque ces 2 hybrides sont débarrassés de leur épis à la récolte, les productions laitières chutent relativement peu. Ces essais ont malheureusement été réalisés avec des niveaux de production faibles (15 à $17 \mathrm{~kg}$ de lait par vache et par jour) et les teneurs en matière sèche des ensilages sans épis sont faibles par rapport à celles des ensilages normaux (24\% contre $34 \%$ ), introduisant un biais important par les quantités ingérées. Rémond et Journet (1977) montrent, quant à eux, que des maïs pauvres en grain (20 à $30 \%$ de grain dans la matière 
sèche) à la suite de stress hydriques conduisent à des productions laitières inférieures de 1,7 à $2,4 \mathrm{~kg}$ à celles permises par des ensilages normaux (45\% grain) mais ces deux types d'ensilage n'ont pas été testés simultanément et les apports de complément n'étaient pas parfaitement comparables. Enfin des comparaisons de variétés ou de densités de semis, faites par l'ITEB (1988-89-90), montrent que les ensilages les plus pauvres en grain ont été les moins bien valorisés alors qu'une expérimentation menée à l'INRA de Lusignan, comparant deux hybrides différents, montre que l'un des génotypes, bien que plus pauvre en grain, est mieux utilisé que l'autre (Barrière et Emile 1990). En fait, dans toutes ces comparaisons, la teneur en grain n'est pas le seul facteur de variation entre les régimes testés et, en particulier, les parties non-grain des différents ensilages ne sont pas comparables (densité de semis, stress en végétation ou génotypes).

Le but des essais présentés ici est de comparer des ensilages provenant du même génotype, de la même parcelle, avec des caractéristiques des parties non-grains comparables et qui ne se différencieraient donc que par leur teneur en grain. Nous avons pour cela retiré des épis dans la parcelle au moment de la récolte afin de diminuer la teneur en grain tout en ne modifiant pas la quantité ni la qualité du non-grain. Cette méthode a déjà été employée par Andrieu et Demarquilly (1974) pour appauvrir ou enrichir des ensilages distribués à des moutons en mesures de digestibilité. Les ensilages ainsi réalisés (appelés "normaux" et "appauvris") ont été distribués à des vaches laitières. La comparaison a été effectuée durant 3 années avec des niveaux d'apport de concentrés conformes aux recommandations habituelles, puis une quatrième année avec des apports de concentré volontairement plus faibles.

\section{L'effet de la teneur en grain de l'ensilage a été étudié en utilisant la même parcelle de maïs, dont une partie a été récoltée après élimination d'un épi sur quatre ou sur huit.}

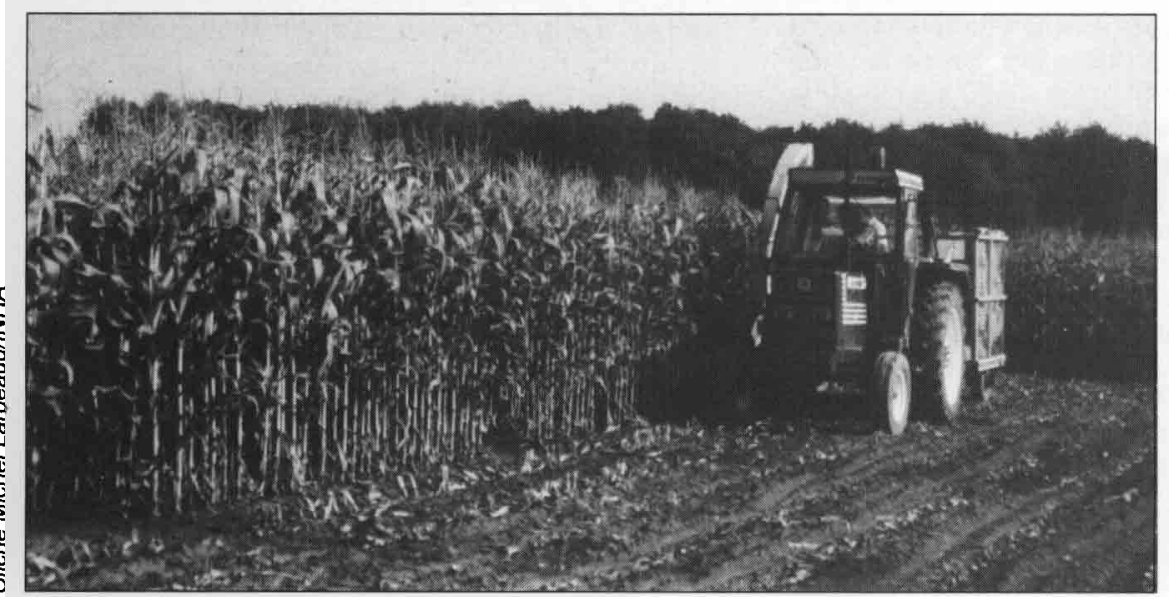

\section{1 / Principales conditions expérimentales}

Implantées à l'INRA de Lusignan (Vienne), les parcelles de maïs ont été conduites dans les conditions courantes des exploitations modernes. Une partie de la parcelle a été ensilée en plante entière. L'autre partie a été récoltée 24 à 48 heures plus tard après élimination d'1 épi sur 8 (essai 1) ou d'1 épi sur 4 (essais 2,3 et 4 ). Le maïs "appauvri" ainsi obtenu se caractérisait donc par une teneur en grain plus faible que celle du fourrage "normal" mais les parties non-grain étaient directement comparables. Le léger décalage de récolte entre les deux parties de la parcelle a réduit les différences de teneur en matière sèche liées au retrait d'une partie des épis. Les variétés de maïs utilisées étaient Dea (essais 1, 2 et 4 ) et Arcade (essai 3). La première, très cultivée, est un hybride inscrit en 1980 sur des critères grain avant la mise en place de la mention ensilage ; la seconde, de même précocité, est inscrite avec seulement la mention ensilage (1986).

Ces ensilages ont été distribués à des vaches laitières. Les principales conditions expérimentales sont détaillées dans le tableau 1. Le troupeau expérimental de 55 vaches FFPN x Holstein d'un niveau moyen de production de $7500 \mathrm{~kg}$ de lait par an environ a permis chaque année de constituer des lots comparables ( 8 à 10 animaux dont 2 à 4 primipares) selon les critères habituels de mise en lots et prenant en compte les performances réalisées pendant la pré-expérience (peu après le pic de lactation).

Les essais se sont déroulés durant 11 à 14 semaines en hiver. Chaque lot d'animaux a reçu comme fourrage de base l'un des 2 ensilages effectués à l'automne précédent, normal ou appauvri, constituant ainsi les 2 traitements expérimentaux. Ces ensilages de maïs, distribués à volonté (5 à $10 \%$ de refus) ont été équilibrés, par les mêmes apports de tourteaux tannés, d'urée et d'un concentré minéral vitaminé. Un concentré de production, type tourteau de soja-céréales, a été distribué en fonction des productions laitières attendues. Pour les trois premiers essais, les quantités de concentrés apportées ont été conformes aux recommandations INRA (1988). Lors du quatrième essai, nous avons réduit les apports de concentrés de façon à placer les animaux en état de sous-alimentation énergétique tout en veillant à ne pas induire de déficit azoté. Nous avons alors ajouté un troisième lot, témoin positif, recevant l'ensilage "normal" avec une complémentation habituelle (Lot "Témoin").

Les mesures individuelles ont porté sur les quantités ingérées (fourrages et concentrés), la production et la composition du lait ainsi que sur les variations de poids des animaux, corrigées des variations de poids du contenu digestif $(4 \mathrm{~kg} / \mathrm{kg}$ de matière sèche ingérée ; Chilliard et al 1987). Des bilans énergétiques et azotés ont été réalisés par différence entre 
Tableau 1. Principales conditions expérimentales

\section{Le déroulement \\ Préexpérience de 2 à 3 semaines \\ Période expérimentale de 11 à 14 semaines \\ Les lots}

24 à 30 vaches Prim'Holstein, appariées en 3 lots selon les critères habituels, après le pic de lactation.

\section{Essais}

Nombre d'animaux par lot (dont primipares)

Caractéristiques moyennes des lots à la mise en lots :

\section{lait $(\mathrm{kg} / \mathrm{j})$}

taux butyreux $(\mathrm{g} / \mathrm{kg})$

taux protéique $(\mathrm{g} / \mathrm{kg})$

poids vif $(\mathrm{kg})$

En début d'essai :

stade de lactation (j)

\section{1}

8

(2)

29,5
42,6
29,3
582

107

$\begin{array}{ccl}2 & 3 & 4 \\ 10 & 10 & 10 \\ (2) & (4) & (3)\end{array}$

\section{La ration}

Ensilage de maïs distribué à volonté

Complément de correction composé de tourteau tanné et urée

Complément minéral ( $12 \% \mathrm{P} ; 16 \% \mathrm{Ca} ; 5 \% \mathrm{Mg}$ ) enrichi en vitamines

Concentré de production équilibré fermier ou industriel à raison de $1 \mathrm{~kg}$ brut par $3 \mathrm{~kg}$ de

lait au-dessus de la production supposée couverte par la ration de base équilibrée $(22 \mathrm{~kg}$ et

$17 \mathrm{~kg}$ respectivement pour les multipares et primipares). Quantités distribuées selon un schéma prédéterminé prévoyant une persistance laitière hebdomadaire de $97,5 \%$ pour les multipares (98\% pour les primipares).

\section{Conditions d'élevage et d'alimentation}

Stabulation libre sur aire paillée

Salle de traite en épis $(2 \times 4)$. Traites à 7 h 30 et 16 h 30 .

Alimentation dans auges individuelles munies de portillons électro-mécaniques.

Distribution de l'ensilage en 1 fois par jour $(10 \mathrm{~h})$ avec incorporation grossière du concentré correcteur, de l'urée et du CMV. Apport du concentré de production en salle de traite par distributeur automatique.

Les mesures

Elles sont individuelles et concernent :

- l'ingestion de fourrages. Pesées des quantités offertes et refusées et détermination des matières sèches 4 jours/semaine.

- l'ingestion de concentrés (DAC et à l'auge) chaque jour.

- la production laitière. Données quotidiennes (sauf essai $1: 4$ jours par semaine). Composition du lait 2 jours par semaine.

- les pesées des animaux toutes les 3 à 4 semaines. Doubles pesées en début et fin d'essai. Analyses de variance-covariance multivariables, avec prise en compte des données de préexpériences. Calculs effectués avec le programme Anvarm de la bibliothèque Amance (Bachacou et al 1981).

Les données présentées sont ajustées des effets du modèle.

les besoins des animaux (entretien, production de lait, variation de poids corrigé) et les apports de la ration (fourrages et concentrés) en tenant compte des interactions négatives entre fourrages et concentrés liées à l'utilisation de l'énergie (Faverdin et al 1987). Pour ces bilans, destinés à caractériser le niveau de satisfaction des besoins énergétiques et azotés, nous avons admis pour valeurs énergé- tiques des ensilages de maïs, celles fournies par les mesures in vivo. Nous avons dans un second temps supposé l'équilibre entre les besoins et les apports et déduit ainsi les valeurs énergétiques (appelées UFL vaches) auxquelles ont été valorisés chacun des ensilages.

Chaque ensilage a par ailleurs été distribué, à volonté, à 6 moutons en cages à digestibilité 
recevant par ailleurs un correcteur d'ensilage apportant urée, minéraux et vitamines (15 g d'urée/kg de MS). Les coefficients d'utilisation digestive de la matière organique et de la cellulose brute (respectivement notés CUD MO et CUD Cellulose dans les tableaux) ont été mesurés par collecte totale des fèces pendant 5 jours de mesure et ont permis de calculer la valeur énergétique de référence, notée UFL moutons (Andrieu et Demarquilly 1987). Des mesures de digestibilité in vitro par les méthodes jus de rumen (Tilley et Terry 1963) et de solubilité enzymatique APC (Lila et al 1986) ont également été effectuées sur ces ensilages. Enfin, les valeurs énergétiques ont été estimées, à partir des teneurs en grain à la récolte ou en cellulose brute des ensilages (Andrieu 1984) et notées UFL grain et UFL Cellulose. Les teneurs en matière sèche des ensilages ont été corrigées des pertes de produits volatils à l'étuve (Dulphy et Demarquilly 1981).

Les rations à base d'ensilages "normaux" ou "appauvris" ont été distribuées à des vaches laitières. Les mesures ont porté sur les quantités ingérées, les production et composition du lait et le poids vif des animaux

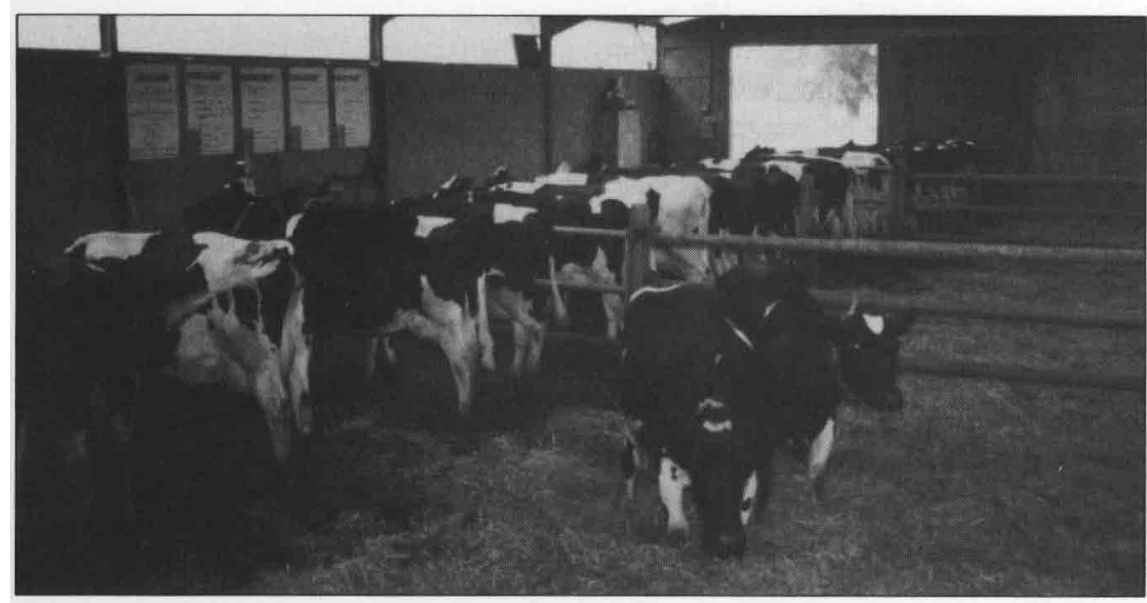

\section{2 / Résultats}

\section{1 / Caractérisation des ensilages et estimation indirecte de leur valeur énergétique}

Le tableau 2 donne les principales caractéristiques des ensilages distribués et leurs valeurs énergétiques, calculées ou estimées selon diverses méthodes.

L'appauvrissement en épis s'est traduit en moyenne des 4 essais par une baisse de la teneur en grain de près de 6 points, soit $12 \%$, et une augmentation de la teneur en cellulose brute de l'ordre de 2 points. Les estimations de la valeur énergétique de l'ensilage appauvri par les teneurs en grain ou en cellulose reflètent nécessairement ces différences de composition (- 0,03 UFL/kg MS par rapport à l'ensilage normal). La digestibilité de la matière organique est inférieure de 2,5 points pour les ensilages appauvris $(68,1 \%$ contre $70,6 \%$ ) ce qui induit une différence de valeur énergétique de 0,06 UFL. La cellulose brute de ces ensilages apparait par contre mieux valorisée $(+2,5$ points $)$.

Les mesures in vitro confirment elles-aussi la meilleure valeur énergétique des ensilages normaux, si l'on fait abstraction des valeurs de digestibilité jus de rumen et APC inexplicables de l'essai 1.

\section{2 / Performances zootechniques et estimation directe de la valeur énergétique des ensilages}

Les résultats présentés dans le tableau 3 se limitent aux données globales de chaque traitement sans séparer les vaches multipares et primipares en raison des très faibles différences de comportement entre le lot et les seules multipares.

Nous présentons d'abord les résultats des trois premiers essais, comparables car conduits à apports énergétiques suffisants, avant d'examiner les données du quatrième essai où ces apports énergétiques ont été réduits.

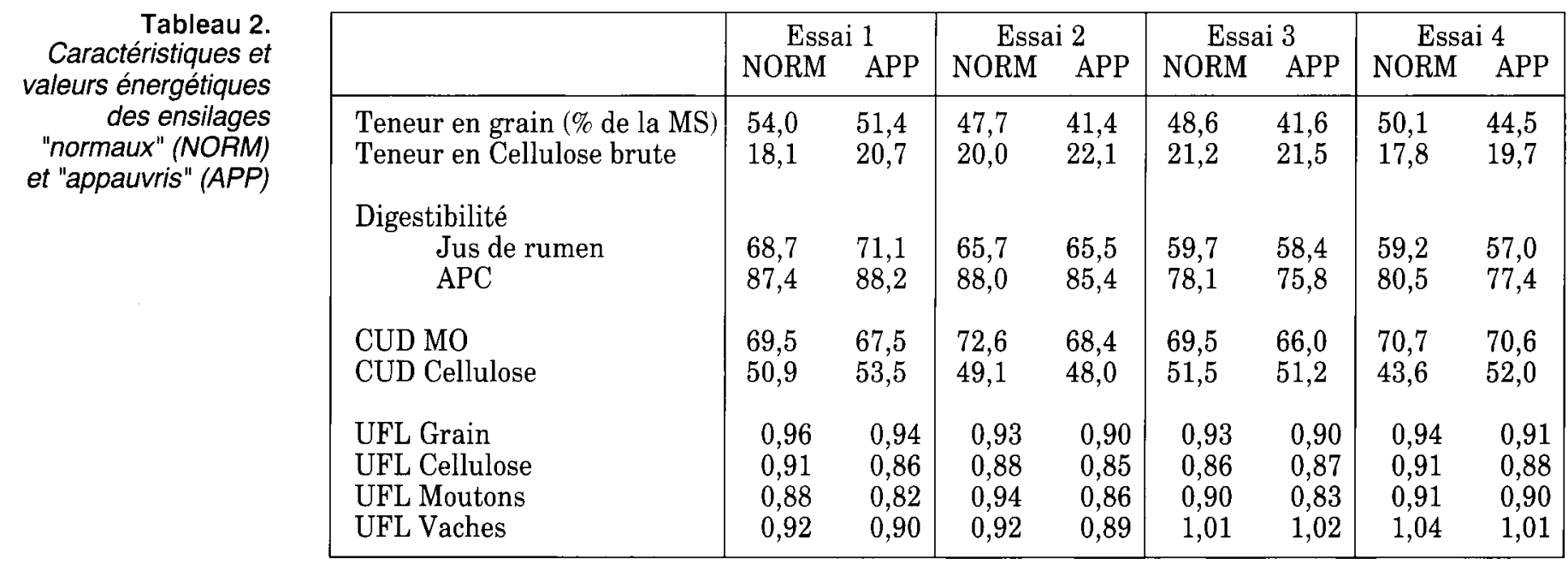


Tableau 3. Performances zootechniques moyennes des animaux des lots normaux (NORM), appauvris (APP) et témoin (TEMOIN). Données par essai et regroupement des essais 1, 2 et 3.

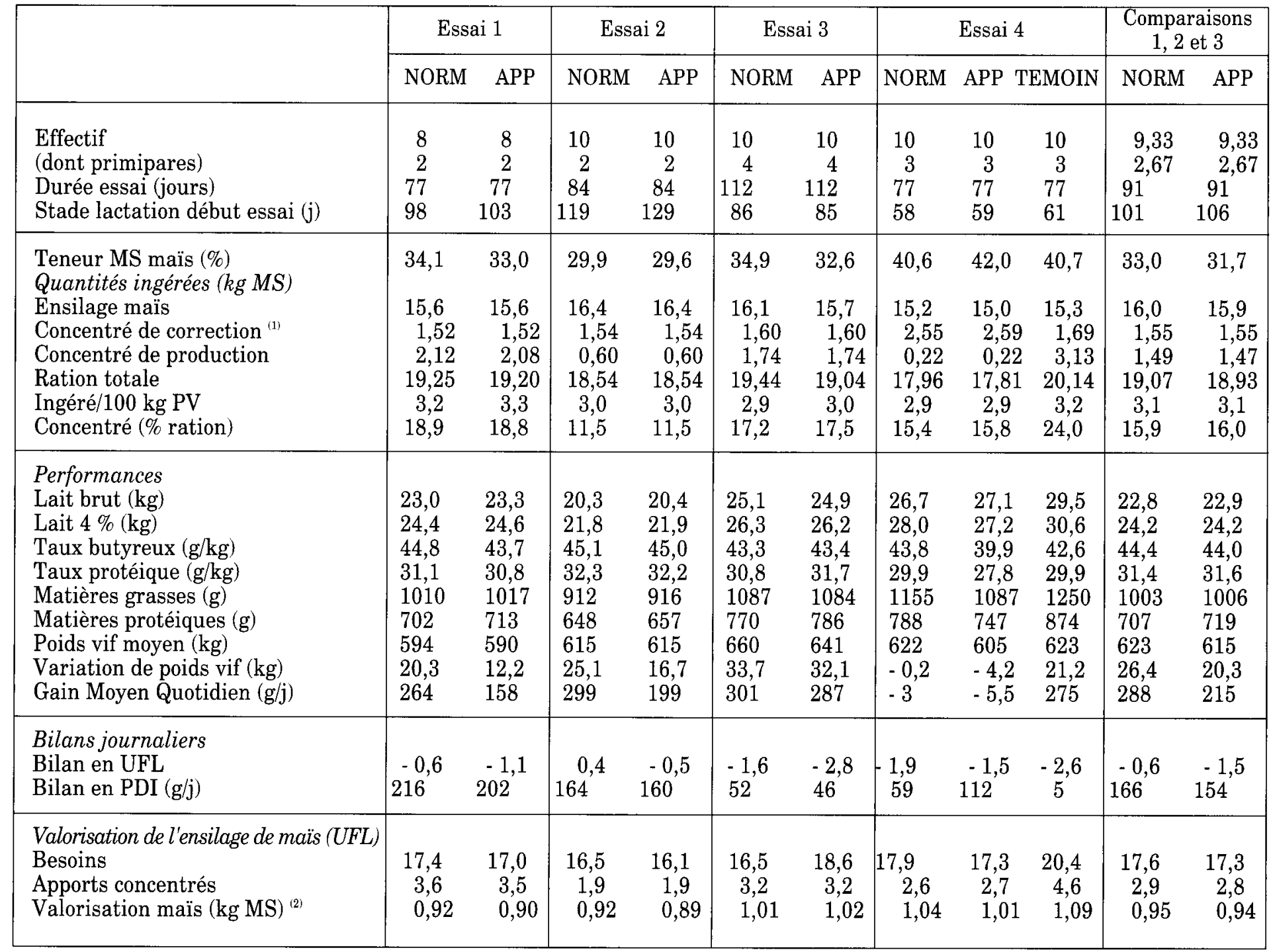

(1) y compris les apports d'urée et de complément minéral vitaminé

(2) (besoins - apports concentrés + interaction) / quantité d'ensilage ingérée

\section{- Effets de la réduction de la teneur en grain en conditions de satisfaction des besoins énergétiques des animaux.}

Avec des proportions de concentrés assez faibles (de 12 à $19 \%$ de la matière sèche totale selon les années), les ingestions de maïs ont été élevées : 16,0 kg pour l'ensemble des 56 vaches avec $16,8 \mathrm{~kg}$ et $14,0 \mathrm{~kg}$ respectivement pour les multipares et primipares. Les quantités ingérées totales ( $3,1 \mathrm{~kg}$ MS pour $100 \mathrm{~kg}$ de poids vif) sont légèrement plus élevées que les valeurs des tables INRA (1988). Dans chaque essai, l'ingestion de l'ensilage appauvri a été comparable à celle de l'ensilage normal (respectivement 15,9 et $16,0 \mathrm{~kg}$ de matière sèche) et ceci malgré une teneur en matière sèche régulièrement inférieure $(31,7$ et $33,0 \%$ ). Les teneurs en matière sèche des ensilages distribués sont satisfaisantes (30 à $35 \%$ en moyenne) mais recouvrent de fortes variations journalières.
Les productions obtenues chaque année, lors des périodes expérimentales, 20 à $25 \mathrm{~kg}$ de lait brut avec des taux butyreux (TB) et protéiques (TP) moyens proches de 44 et $31 \mathrm{~g}$ par $\mathrm{kg}$ reflètent bien les performances habituelles du troupeau, compte tenu des stades de lactation. Tout comme pour les quantités ingérées, on ne constate pas de différence significative ni pour les productions laitières brutes ni pour les productions standardisées (lait à $4 \%$ ) entre les lots ayant reçu l'ensilage appauvri ou normal du même génotype et ceci chacune des trois années. Les vaches des lots "appauvris" ont produit en moyenne $22,9 \mathrm{~kg}$ de lait par jour à $44,0 \mathrm{~g} / \mathrm{kg}$ de taux butyreux et $31,6 \mathrm{~g} / \mathrm{kg}$ de taux protéique, les lots "normaux" produisant $22,8 \mathrm{~kg}$ de lait à $44,4 \mathrm{~g} / \mathrm{kg}$ et $31,4 \mathrm{~g} / \mathrm{kg}$.

En tenant compte des variations de contenu digestif, les animaux ont globalement repris du poids chaque année $(211,249$ et $294 \mathrm{~g} / \mathrm{jour}$
La teneur en grain de l'ensilage de maïs n'a pas eu d'effet sur les quantités ingérées ni sur la production et la composition du lait. 
pour les 3 essais). La reprise de poids a toutefois été plus faible chaque année dans les lots appauvris (215 g/jour) par rapport aux lots normaux ( $288 \mathrm{~g} / \mathrm{jour}$ ).

Les bilans azotés des lots montrent qu'en moyenne les apports sont supérieurs de $10 \%$ aux besoins. Remarquons toutefois que les reprises de poids n'intervenant pas dans ces bilans, ces besoins se trouvent donc sous-estimés. Les bilans énergétiques, établis en prenant pour valeur énergétique des ensilages les données de référence obtenues in vivo sur moutons standards et intégrant les variations de poids, sont légèrement négatifs $(-1,5$ et - 0,6 UFL pour les lots appauvris et normaux) ce qui montre que nos animaux n'étaient pas en suralimentation. Les valeurs de valorisation énergétique de ces ensilages par les vaches laitières sont relativement variables entre années $(0,91,0,90$ puis $1,01 \mathrm{UFL})$ mais très proches entre les deux traitements $(0,94$ et 0,95 UFL pour les lots appauvris et normaux). Si l'on examine les variations individuelles de ces valeurs de valorisation, on peut observer que, parmi les animaux valorisant très bien l'ensilage reçu, ceux qui consomment les ensilages appauvris ont plus tendance à le valoriser efficacement en lait alors que les autres se caractérisent par des reprises de poids plus importantes.

\section{- Effets de la réduction de la teneur en grain en conditions de sous alimentation énergétique}

Lors du 4ème essai, les animaux en expérimentation sont moins avancés en lactation, ce qui explique les proportions de concentrés plus élevées que les années précédentes ainsi que les productions laitières elles aussi supérieures.

Les ensilages récoltés se sont caractérisés par une teneur en matière sèche élevée et une inversion de classement entre les teneurs de l'ensilage appauvri et celui du normal $(42,0$ et $40,6 \%$ ) en raison des conditions très desséchantes de l'été 1989 au moment de la récolte.

Par rapport au lot témoin conduit de façon classique ( $24 \%$ de concentrés) et comparable aux 3 essais antérieurs, les deux lots expérimentaux ont consommé une ration moins riche en concentrés $(15 \%)$ avec en moyenne $2,06 \mathrm{~kg}$ de MS de concentrés en moins (soit $0,85 \mathrm{~kg}$ de tourteau tanné en plus et $3,13 \mathrm{~kg}$ de concentré de production en moins). Les quantités de maïs ingérées relativement faibles en raison peut être des teneurs en matière sèche trop élevées ont été cependant comparables entre lots et nous n'avons pas observé de substitution entre fourrages et concentrés. Les performances zootechniques de ces deux lots expérimentaux sont globalement moindres que celles du témoin, tant en ce qui concerne les productions de lait $(-2,5 \mathrm{~kg}$ de lait brut et $-3,1 \mathrm{~kg}$ de lait à $4 \%$ ) que les reprises de poids $(+2 \mathrm{~kg}$ contre $+21 \mathrm{~kg})$.

Le lot appauvri a produit $0,4 \mathrm{~kg}$ de lait brut de plus que le lot normal (27,1 contre $26,7 \mathrm{~kg})$ mais avec une très forte dégradation des taux ( -4 points de taux butyreux et -2 points de taux protéique) pendant les 10 semaines d'essai. Ceci explique, avec des quantités ingérées proches, que le bilan énergétique du lot appauvri soit un peu moins négatif que celui $\mathrm{du}$ lot normal (respectivement - 1,5 et $-1,9$ UFL). Les variations de poids des animaux des 2 lots ont été faibles (perte de poids de $4,2 \mathrm{~kg}$ pour l'appauvri et de $0,2 \mathrm{~kg}$ pour le normal sur les 91 jours d'essai). Les valeurs de valorisation, dans ces conditions draconiennes, sont élevées mais là encore assez proches (1,01 et 1,04 UFL).

\section{Discussion}

La réduction de 6 points de la proportion de grain dans l'ensilage de maïs, sans compensation par l'augmentation des quantités ingérées, induisant ainsi une baisse des apports quotidiens d'énergie de 1,23 UFL par animal, aurait dû se traduire par une baisse de production de lait à $4 \%$ de plus de $1,0 \mathrm{~kg}$ et d'une chute du taux protéique de 0,8 point ou par une mobilisation des réserves corporelles de l'ordre de $30 \mathrm{~kg}$ sur la période expérimentale (Faverdin et al 1987, Coulon et Rémond 1991).

La comparaison, durant ces essais, d'ensilages normaux ou appauvris en épis montre qu'il n'y a eu d'effet direct de la teneur en grain ni sur les quantités ingérées ni sur la production et la composition du lait. Les reprises de poids des animaux ayant reçu les maïs appauvris ont tendance à être légèrement plus faibles. Les valeurs de valorisation (UFL vaches) élevées que nous avons obtenues peuvent amener à discuter certaines données et en particulier les variations de poids vif et de contenus digestifs et les valeurs énergétiques correspondantes. C'est pourquoi, il convient de s'intéresser ici à l'écart entre les valeurs de valorisation. Cet écart est faible $(0,01 \mathrm{UFL})$, les deux types d'ensilages ont donc été valorisés comme s'ils avaient la même valeur énergétique.

Lors du 4ème essai, avec des apports d'énergie volontairement réduits, l'appauvrissement s'est traduit par une chute importante des taux butyreux et azotés des laits mais aussi par un maintien de la production de lait brut, tout du moins dans la durée de l'essai. Nous n'avons pu noter d'éventuels effets sur la reproduction des animaux.

Ainsi, avec une ration pourtant relativement pauvre en concentrés, une réduction de la teneur en grain de l'ensilage de 50 à $44 \%$ a conduit à une aussi bonne valorisation globale de la ration, en raison probablement d'une amélioration de l'utilisation digestive de la partie non grain. A partir des valeurs de valorisation de la ration et en supposant que la partie grain avait une digestibilité identique dans les deux modalités, la partie non grain des maîs appauvris apparaît ainsi valorisée à 
0,71 UFL alors que dans les ensilages normaux elle ne le serait qu'à 0,66 UFL.

Ces observations rejoignent celles de El Shazy et al (1961) et de Henning et al (1980) mettant en évidence les antagonismes entre les flores amilolytiques et cellulolytiques du rumen. Hemken et al (1971) ont signalé que la digestibilité de la cellulose de leurs ensilages sans épis, mesurée in vivo chez des boeufs, a été améliorée de 8 points et elle l'a été de 2,4 points dans notre comparaison (tableau 2 ). De la même façon qu'une notion d'interaction digestive négative entre fourrages et concentrés a été introduite et est intégrée dans les raisonnements du rationnement des vaches laitières (Faverdin et al 1987), il paraît raisonnable de concevoir que dans le cas du maïs, aliment composite, tout se passe comme s'il y avait une interaction digestive négative entre les parties grain et non-grain. Cette interaction serait d'autant plus forte que la proportion de concentrés dans la ration et la teneur en grain de l'ensilage seraient plus élevées.

Ces résultats, même s'ils surprennent au premier abord, doivent rassurer le sélectionneur de maïs ensilage et l'inciter à reporter une partie de ses efforts de l'amélioration de la productivité en grains sur l'amélioration de la productivité totale en biomasse : un accroissement significatif de ce rendement avec une teneur en grain qui resterait correcte sans être exceptionnelle ne présenterait pas de risque zootechnique pour des vaches laitières.

Pour l'éleveur, il paraît certes prudent de s'assurer, en l'état actuel des connaissances, que la teneur en grain de son ensilage ne sera pas inférieure à $40 \%$ mais il ne semble pas utile qu'elle soit notablement supérieure à ce seuil. Barrière et al (1992) ont en effet obtenu des valeurs énergétiques de $0,90 \mathrm{UFL}$ avec des ensilages contenant de 20 à $60 \%$ de grain, pour les extrêmes. Rappelons également que nos essais ont été réalisés sur des cultures bien conduites et que ces ensilages à plus faible teneur en grain (appauvris) ne sont pas comparables à ceux obtenus en situations de stress (semis tardif, densité élevée, sécheresse...).

Ces observations confirment également les difficultés de prédiction de la valeur d'utilisation des fourrages, que ces prédictions soient basées sur la composition chimique ou morphologique, sur des méthodes chimiques, pour lesquelles il semble nécessaire d'avoir un grand nombre de répétitions pour estimer de façon fiable la valeur alimentaire, ou sur des mesures in vivo chez des moutons à l'entretien dont la précision sur 2 mesures a un intervalle de confiance de 0,05 (Barrière et Emile 1990 ; Barrière et al 1992). Il apparaît, en effet, qu'aucune des méthodes utilisées n'a pu prédire correctement les résultats zootechniques obtenus.

Enfin, même si un essai sur chèvres laitières en lactation (Emile et Broqua 1989, données non publiées) semble confirmer ces conclusions, il faut rester prudent quant à leur généralisation à d'autres types de production en raison des différences déjà mises en évidence par de nombreux auteurs (Kilmer et al 1979, Aerts et al 1984, Chenost et MartinRosset 1985, Moran et al 1988, Colucci et al 1989, Dulphy et al 1990). Cette prudence est également fondée sur les résultats d'un essai préliminaire que nous avons mené sur taurillons. Nous y avons noté un ralentissement de la croissance d'animaux consommant un maïs appauvri en épis. Avec cette observation, à préciser par des essais en cours, il se confirme que l'appauvrissement en épis favoriserait ou du moins pénaliserait moins les voies métaboliques orientées vers les productions de lait que celles orientées vers la croissance.

Cette expérimentation, essentiellement conçue comme piste de réflexion pour l'amélioration génétique du maïs, permet d'espérer à moyen terme une augmentation de la productivité de l'hectare de maïs sans perte de qualité et donc une diminution des coûts d'élevage (réduction de la surface fourragère pour un volume de production donné). Ce progrès sera d'autant plus net qu'il sera réalisé simultanément à des améliorations de la digestibilité de la partie non grain (Barrière et al 1992). Les observations tirées de ces essais peuvent également apporter des éléments pour le rationnement quotidien des ruminants au sein de chaque élevage et en particulier sur la stratégie de distribution de fourrages et concentrés dans un contexte de gestion au plus près des dépenses d'élevage.

\section{Remerciements}

Nous tenons particulièrement à remercier Lionel Huguet, Marc Lila et Rémy Traineau pour leurs contributions au suivi des cultures, aux analyses chimiques et aux mesures réalisées sur moutons ainsi que l'ensemble des techniciens qui ont participé à ces expérimentations.

\section{Références bibliographiques}

AERST J.V., DE BOEVER J.L., COTTYN B.G., DE BRABANDER D.L., BUYSSE F.X., 1984. Comparative digestibility of feedstuffs by sheep and cows. Anim. Feed Sci.and Techn. 12(1), 47-56.

ANDRIEU J., 1984. Prévision de la digestibilité et de la valeur énergétique des ensilages de maïs à partir de la composition de la plante à la mise en silos. Bull. Tech. C.R.Z.V. Theix, I.N.R.A., 56, 5-8,

ANDRIEU J, DEMARQUILLY C, 1974. Valeur alimentaire du maîs fourrage. II. Influence du stade de végéta. tion, de la variété, du peuplement, de l'enrichissement en épis et de l'addition d'urée sur la digestibilité et l'ingestibilité de l'ensilage de maïs. Ann Zootech. 23, 1-25.

ANDRIEU J., DEMARQUILLY C., 1987. Valeur nutritive des fourrages : tables et prévisions. Bull. Tech. C.R.Z.V. Theix, I.N.R.A., 70, 61-73.

BARRIERE Y., GALLAIS A., DERIEUX M., PANOUILLE A., 1987. Etude de la valeur agronomique au stade de récolte ensilage de différentes variétes de maïs grain sélectionnées entre 1950 et 1980 . Agronomie, $7,73-79$. 
BARRIERE Y., EMILE J. C., 1990. Effets des teneurs en grain et de la variabilité génétique sur la valeur énergétique du maïs ensilage mesurée par des vaches laitières. Agronomie, 10, 201-212.

BARRIERE Y., TRAINEAU R., EMILE J.C., HEBERT Y, 1992. Variation and covariation of silage maize digestibility estimated from digestion trials with sheeps. Euphytica, (in press).

CHENOS'T M MARTIN-ROSSET W 1985. Comparaison entre espèces (mouton, cheval, bovin) de la digestibilité et des quantités ingérées de fourrages verts. Ann. Zootech. $3: 291-312$.

CHILLIARD Y., REMOND B., AGABRIEL J., ROBELIN J., VERITE R., 1987. Variations du contenu digestif et des réserves corporelles au cours du cycle gestation lactation. Bull. Tech. C.R.Z.V. Theix, I.N.R.A., 70, 117-131.

COLUCCI P.E., MACLEOD G.K., GROVUM W.L., CAHILL L.W., Mc MILLAN I., 1989. Comparative digestion in sheep and cattle fed different forage to concentrate ratios at high and low intakes. J. Dairy Sci., 72 : 1774-1785.

COULON J B., REMOND B., 1991. Réponses de la production et de la composition du lait de vache aux variations d'apports nutritifs. INRA, Prod. Anim., 4, 1, 49-56.

DULPHY J.P., DEMARQUILLY C., 1981. Problèmes particuliers aux ensilages. in C. Demarquilly éd. : "Prévision de la valeur nutritive des aliments des ruminants". INRA Publications, Paris, p. 81-104.

DULPHY J.P., CARLE B., DEMARQUILLY C., 1990. Quantités ingérées et activités alimentaires comparées des ovins, bovins et caprins recevant des fourrages conservés avec ou sans aliment concentré. I. Etude descriptive. Ann. Zootech., 39, 95-111.

EL SHAZY K., DEHORITY B.A., JOHNSON R.R., 1961. Effect of starch on the digestion of cellulose in vitro and in vivo by rumen micro-organisms. J. Anim. Sci., 20,268273
FAVERDIN P., HODEN A., COULON J.B., 1987. Recommandations alimentaires pour les vaches laitières. Bull. Tech. CRZV Theix, INRA, 70, 133-152.

HEMKEN R.W., CLARCK N.A., GOERING H.K., VANDERSALL J.H., 1971. Nutritive value of corn silage as influenced by grain content. J. Dairy Sci., 54, 383-389.

HENNING P.A., VAN DER LINDEN Y., MATTHEYSE M.E., NAUHAUS W.K., SCHWARTZ H.M., 1980. Factors affecting the intake and digestion of roughage by sheep fed maize straw supplemented with maize grain. J. Agric. Sci. Camb., 94, 565-573.

INRA, 1988. Alimentation des bovins, ovins et caprins. Ed. R. Jarrige, INRA Publications, Paris.

ITEB, 1988, 1989 et 1990 . Bulletin Ognoas Flash, ARPEB, Domaine d'Ognoas, 40190 Arthez d'Armagnac, $\mathrm{n}^{\circ} 12,14,16,17$.

KILMER L.H., WANGSNESS P.J., KESLER E.M., MULLER L.D., GRIEL L.C., KRABILL L.F., 1979. Voluntary intake and digestibility of legume and grass diets fed to lactating cows and growing wethers. J. Dairy Sci., 62, 1272-1277.

LILA M., BARRIERE Y., TRAINEAU R., 1986. Mise au point et étude d'un test enzymatique de la digestibilité de fourrages pauvres ou riches en amidon. Agronomie, 6 (3) : 285-291.

MORAN J.B., LEMERLE C., TRIGG T.E., 1988. The intake and digestion of maize silage-based diets by dairy cows and sheep. Anim. Feed Sci. and Techn. 20 : 299-312. REMOND B., JOURNET M., 1977. Utilisation de 2 ensilages de maïs de composition morphologique différente par les vaches laitières. Bull. Tech. C.R.Z.V. Theix, I.N.R.A., 29, 49-52.

TILLEY J.M.A., TERRY R.A., 1963. A two stage technique for the in vitro digestion of forage crops. J. Br. Grassl. Soc., 18, 104-111.

\section{Summary}

\section{Maize silage for dairy cows : effect of grain content on performances}

The effects of a lower grain content in maize silage hybrids (resulting from discarding a part of the ears on harvest time) on DM intake, body weight variations, yield and composition of milk by highyielding dairy cows were investigated. Cows were individually fed ad libitum with the experimental or the control roughage diets. They received concentrates according to requirements ( 3 trials) or under energetic requirements ( 1 trial). The experimental periods lasted for 10 to 14 weeks. Neither roughage DM intake $(15.9 \mathrm{~kg} / \mathrm{cow} / \mathrm{day}$ for the experimental diet versus $16.0 \mathrm{~kg}$ for the control) nor body weight gain $(20 \mathrm{~kg} / \mathrm{cow} /$ period versus $26 \mathrm{~kg}$ ) or milk yield $(22.9 \mathrm{~kg} /$ cow/day versus $22.8 \mathrm{~kg}$ ) were modified by the lowering of the grain level from $50 \%$ (total DM basis) to $44 \%$. Milk fat concentration and protein content were only affected by the experimental diet during the last trial. The apparent digestibility of the non-grain part of the silage, estimated by cows performances, seemed to be higher when the grain content is lower (1.21 Mcal/DM kg versus $1,12 \mathrm{Mcal})$. None of the digestibility predicting tests used in this study was pertinent for the prediction of the energetic value, according to cows performances. Maize hybrids with a higher total DM yield but with a lower grain content ( 5 to 10 points) than the modern hybrids could be fit for dairy cows feeding.

EMILE J.C., BARRIERE Y., 1992. Effets de la teneur en grain de l'ensilage de maïs sur les performances zootechniques de vaches laitières. INRA Prod. Anim., 5 (2), 113 120 . 